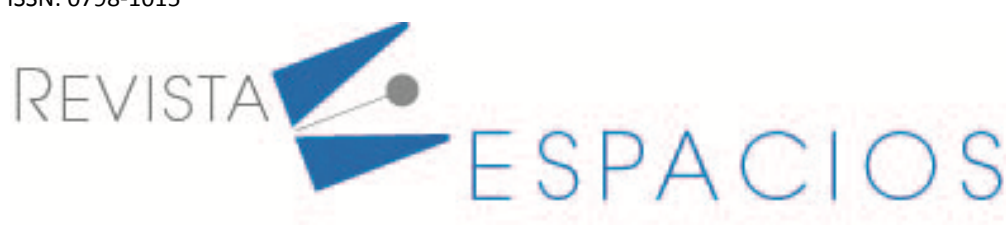

\title{
Reconocimiento de perfiles virtuales falsos en clases educativas
}

\section{Recognition of fake virtual profiles in educational classes}

\author{
SUAREZ CASTRILLON, Albert M. ${ }^{1}$ \\ RINCON, Isbelia K. ${ }^{2}$ \\ SUAREZ, Sir A. ${ }^{3}$
}

\begin{abstract}
Resumen
La importancia de esta investigación se origina del uso de videoconferencias en la educación, como alternativa para continuar con la enseñanza después de los problemas ocasionados por el COVID-19. Permitiendo detectar cuando una persona forma parte del curso o ingresó de forma indebida, el cual podría orientar a realizar acciones indebidas. El reconocimiento se obtiene de los descriptores de textura extraídos de las imágenes, con una tasa de error del 7,64\% en imágenes modificadas y sin errores en clases en línea.

Palabras clave: descriptores de textura, reconocimiento facial, COVID-19, clases virtuales

Abstract

The importance of the research stems from the use of video conferencing in education, as an alternative to continue teaching, after the problems caused by COVID-19. Allowing to detect when a person is part of the course or entered improperly, which could lead to improper actions. The recognition is obtained from the texture descriptors extracted from the images, with an error rate of $7.64 \%$ in modified images and without errors in online classes.

key words: texture descriptors, facial recognition, COVID-19, virtual classes.
\end{abstract}

\section{Introducción}

Las clases presenciales han tenido que migrar a clases en línea como consecuencia del COVID-19, utilizando el soporte que ofrece cada institución educativa, el cual ha terminado por aislar a todos los estudiantes de sus instituciones. Para el mes de octubre de 2020 las cifras a nivel mundial superan el millón de muertos y 37,5 millones de contagios (AFP, 2020). La educación durante el COVID-19 presenta retos y oportunidades con una forma de atender y relacionarse entre docentes y estudiantes, y las investigaciones en todas las áreas dan una muestra de ello. En medicina se plantean la posibilidad de utilizar videos que van de 6 a 15 minutos y que cubren 1 o 2 temas junto a documentación de lectura (Alemán, Vera, y Patiño-Torres, 2020); en el Foro Iberoamericano de educación Medica analizan las propuestas de cada país, encontrando similitud en el uso de plataformas educativas y videoconferencias (Núñez-Cortés, Reussi, García Dieguez, y Falasco, 2020). La Sociedad Española de

\footnotetext{
${ }^{1}$ Docente. Programa Ingeniería Mecánica. Universidad de Pamplona. Grupo de Investigación Gimup. albertmiyer@unipamplona.edu.co

${ }^{2}$ Docente. Programa Ingeniería de Sistemas. Universidad Francisco de Paula Santander Ocaña. Grupo de Investigación Grucite. ikrinconp@ufpso.edu.co

${ }^{3}$ Docente. Programa Ingeniería de Sistemas. Universidad Francisco de Paula Santander Ocaña. Grupo de Investigación Grucite. sasuarezc@ufpso.edu.co
} 
Cardiología presenta en consenso el uso de consultas telemáticas con la finalidad de reducir el riesgo de contagio y recoge los criterios para su adecuado funcionamiento (Barrios et al., 2020), mientras que Núñez-Cortés (2020) realiza un extenso trabajo de la educación médica en plena pandemia, abordando temas como los podcast y la realidad virtual. No solo es impartir una clase, también los trabajos presentan la responsabilidad que se debe tener con el medio ambiente, como un todo, donde la casa, el trabajo y la educación deben formar un conjunto que permita saltar los obstáculos presentes en la educación (Ruiz, 2020). Al realizar un análisis de los recursos utilizados por cada país, en Sur América (BID, 2020) se llega a la conclusión que todos utilizan contenido digital, solo Brasil y Uruguay tenían una implementación a nivel nacional con plataformas de aprendizaje, y el material físico enviados por sistemas de transporte nacional no son utilizadas en Bolivia, Brasil, Uruguay y Venezuela; mientras que la televisión y la radio son medios bastante utilizados por su naturaleza de tener contenido inmediato y a bajo costo.; ninguno de los países mantienen las escuelas abiertas, llamando la atención Nicaragua, un país centro americano que no muestra medidas de prevención y aislamiento educativo, por lo tanto no utiliza ninguna herramienta tecnológica; mientras Colombia tiene un masivo uso de todas las tecnologías y se muestra un factor común en el uso de contenido digital, TV o radio. Antes de iniciar las propuestas de educación los países de América Latina tuvieron que analizar los recursos existentes, donde los sistemas de educación y gestión educativa eran factores a revisar. Para el momento del inicio de la pandemia los indicadores de Colombia eran muy bajos en materia de tutorías virtuales, con una conectividad en escuelas medio-baja, con niveles aceptables en plataformas digitales y muy buena en paquetes de recursos y repositorios centrales de contenido. El acceso a computadores en los hogares era de solo el $21 \%$, un problema enorme en la transición del modelo educativo presencial al virtual.

Todos los recursos son propuestas válidas y una de ellas son las clases virtuales utilizando plataformas de videollamadas, siendo las que ofrecen posibilidades de conexión síncrona, entre ellas se encuentran Meet (Google, 2020) y Zoom (2020) por su gran posibilidad de adaptación y funcionalidad, los resultados de las plataformas son positivos al obtener una mayor interactividad entre docente-estudiante (Beltrán, 2017; BravoMancero, Larrea, Ruales, y Cruz, 2020; Vidal Martínez y Camarena Gómez, 2015). Las clases virtuales han ingresado a cada uno de los hogares donde estudiantes de todas las edades se conectan con un docente, esto puede contribuir a crímenes digitales o perfiles virtuales falsos los cuales son nocivos y difíciles de detectar para estudiantes en edades tempranas, debido a que toman clases o se reúnen con amigos sin un acompañante mayor, inclusive los mismos docentes no logran reconocer a sus estudiantes debido a la dificultad de concentrarse en el rostro del estudiante en cada conferencia o clase. Este problema se debe a que Las instituciones educativas siguen implementando plataformas educativas virtuales pero pocas han diseñado una propuesta de control de fraude originado por un perfil falso, donde cada docente debe crear una manera de evitarlo si desean que la calidad se mantenga al utilizar la virtualidad. Aunque existen herramientas como Proctorfree, Proctorio o Remote Proctor Now, entre otras, el alto costo de su implementación y el tiempo reducido en que se debió pasar de las clases presenciales a las virtuales han impedido un protocolo adecuado que permita la supervisión de las pruebas que deben presentar los estudiantes y sus restricciones se enfocan más a pruebas de tipo cuestionario, donde impiden el acceso a otros documentos dentro del mismo computador pero todas las pruebas no se basan en este tipo de evaluación. La suplantación de identidades es una de las desventajas de la evaluación en línea, llegando hacer extremadamente alto el fraude en los exámenes; donde los estudiantes han admitido haber cometido fraude en algún momento con porcentajes que van del $60 \%$ al $95 \%$ en países como Israel y Corea del Sur y mantienen patrones de comportamiento similares en el resto de países (Friedman, Blau, y Eshet-Alkalai, 2016) . En Colombia, la Universidad Nacional Abierta y a Distancia (UNAD) es una de las primeras en utilizar un sistema de biometría y videovigilancia para validar la identidad de cada estudiante al momento de la presentación de cada prueba (Cáceres-Piñaloza, 2020); pero hay que resaltar que la UNAD es una institución de educación superior que centra sus registros académicos en cursos virtuales, es por 
eso que mantiene un sistema de verificación de identidades; mientras que las instituciones con educación presencial han pasado directo a la modalidad virtual sin un protocolo adecuado.

Para lograr el reconocimiento de una persona se utiliza la Biometría la cual mediante las características físicas, químicas y de conducta de una persona puede ser identificada sin necesitar una colaboración extremada por parte del estudiante debido a que es un método no invasivo (Alvez et al., 2014). Las características físicas es la que se puede obtener de una forma más rápida y sin el conocimiento del estudiante, ampliamente utilizada en sistemas de vigilancia en aeropuertos, centros comerciales y en general en áreas de seguridad, al captar y procesar el rostro de cada persona. La visión por computador, es una disciplina que incluye métodos que van desde la adquisición hasta la identificación y verificación en diferentes procesos industriales y pueden utilizarse para la comprensión de imágenes del mundo real, es por eso que dentro de las áreas de estudio se encuentra el reconocimiento de rostros, siendo una de las más estudiadas encontrando soluciones apropiadas a diferentes problemas; dentro de ellas se encuentra los estudios realizados al fraude en las pruebas que presentan los estudiantes en plataformas virtuales al realizar un reconocimiento de rostro. Por ejemplo en España se utiliza para evitar el fraude académico (Gámez y Agapito, 2016), utilizando el software Smowl implementado mediante un plugin en la plataforma Moodle, el estudio se basa en como el mecanismo afectaría el rendimiento académico de los estudiantes al saber que estaban siendo monitoreados; estas herramientas son restrictivas debido a tener un único uso en una plataforma determinada. La mayoría de herramientas utilizan métodos basados en técnicas de análisis de imagen como: SIFT (scale-invariant feature transform) que permite detectar características locales de interés en una imagen (Karami, Shehata, y Smith, 2015) y SURF (Speeded Up Robust Features) el cual es basado en el anterior pero disminuye el nivel de procesamiento (Oyallon y Rabin, 2015); ambos pueden ser implementados por los investigadores de tal forma que logren tasas de reconocimientos más altas y no sean vulnerables fácilmente. Muchos de los trabajos intentan fusionar los dos métodos o crear nuevas medidas a partir de ellas que permitan aumentar la tasa de reconocimiento utilizando por ejemplo al SIFT como detector $y$ el SURF para descripción (Vinay, Hebbar, Shekhar, Murthy, y Natarajan, 2015); también se puede aumentar la robustez al agregar las emociones que puede presentar una persona a los descriptores de SIFT (Neeru y Kaur, 2016) y su función es la reducción en el almacenamiento ya que requiere de una sola imagen con diferentes gestos y no diferentes imágenes de la misma persona. Además de las técnicas anteriores también se ha utilizado el patrón binario local (Ali Khan, Hussain, y Usman, 2016) para reconocer la expresión facial que interprete factores presentes en la personalidad. Para evitar ataques se utilizan mascaras de convolución (Jia, Guo, y Xu, 2020) demostrando que los modelos 3D ofrecen mejores resultados que las fotos en 2D. Si se analiza la imagen mediante la textura se puede y utilizar los descriptores de Haralick, los cuales han sido utilizados con resultados de clasificación del 80 y 90 por ciento (Senthilkumar y Gnanamurthy, 2019); la variabilidad de la luz o inclusive de los gestos también son investigados y resueltos con estos descriptores, con un reconocimiento total en videoconferencia con muestras ya entrenadas (Agarwal, Singh, y Vatsa, 2016). Pero también puede utilizarse el sistema biométrico de huella dactilar como ayuda al sistema de reconocimiento (Kushwaha y Nain, 2020) usando el nivel de gris de los descriptores de textura; los descriptores son analizados y verificados probando nuevas técnicas que permiten un reconocimiento adecuado de la persona por medio del rostro al realizar una fusión con distintas técnicas (Alazawi, Shati, y Abbas, 2019; Ding, 2017).

De acuerdo al problema de suplantación de identidades que puede presentarse en las clases realizadas de forma virtual y las herramientas de técnicas de procesamiento de imágenes existentes que son difíciles de implementar, se presenta un método para el reconocimiento del rostro mediante los descriptores de Haralick, que permite reconocer los perfiles virtuales falsos, logrando una reducción de la dimensionalidad y el tiempo de procesamiento. Aunque el método puede ser utilizado para otras tareas similares, solo se ha comprobado su funcionamiento para clases virtuales donde la población no es tan alta. 


\section{Metodología}

El método de reconocimiento usando descriptores de textura, la clasificación y correlación se obtiene de cinco pasos: adquisición de imágenes, preprocesamiento, extracción de características, reconocimiento de patrones y evaluación de resultados (ver Figura 1).

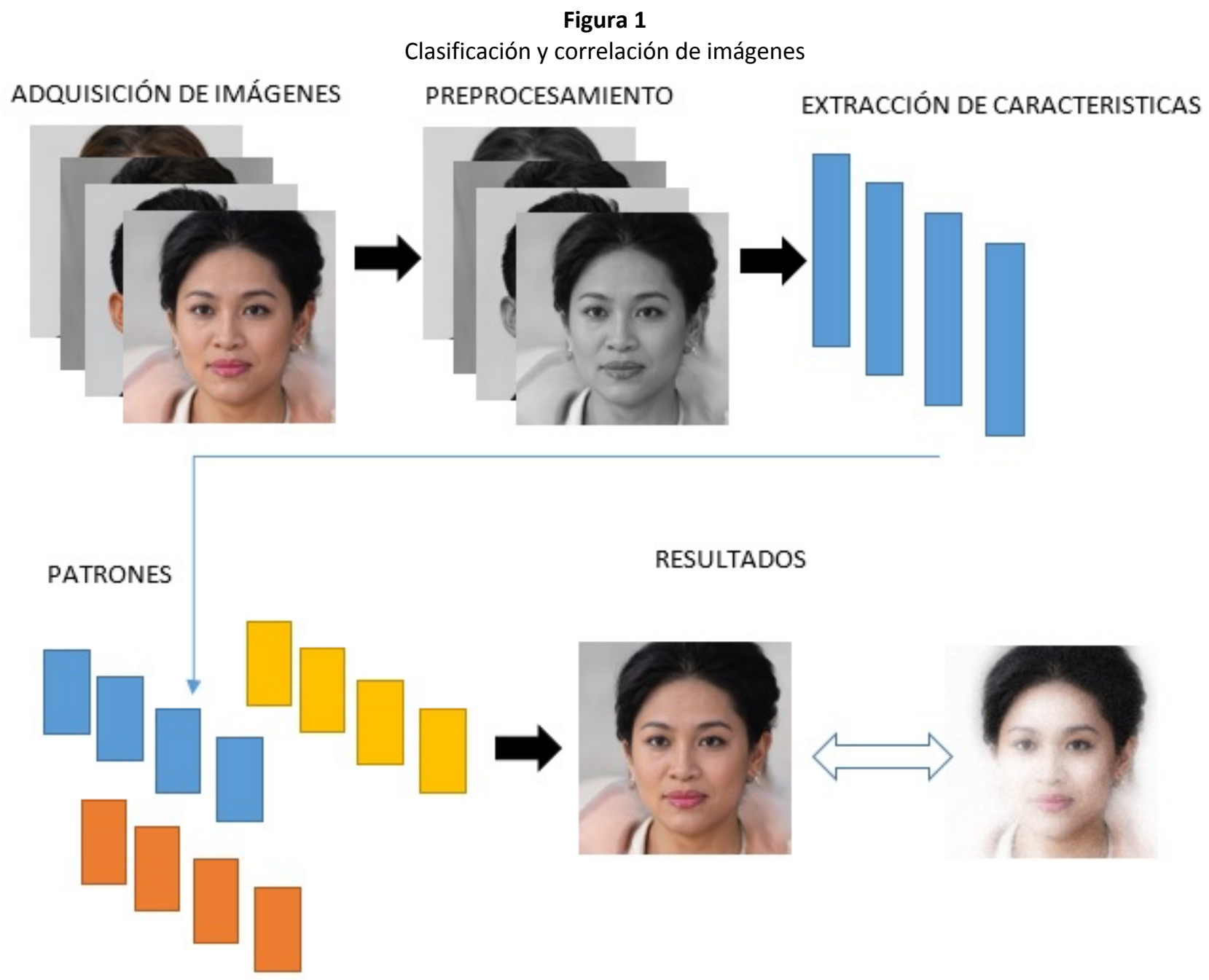

Fuente: elaboración propia utilizando foto de Generated Photos

\subsection{Adquisición de imágenes y preprocesamiento}

En el proceso de adquisición se utilizan 12 imágenes de la base de datos de Generated Photos (2020), las cuales son modificadas para formar un conjunto de 480 imágenes en total, la finalidad era simular ambientes que pueden ocurrir con la iluminación, distorsión en la toma de la imagen, problemas de conexión que envían imágenes con diferentes niveles de rugosidad e inclusive objetos existentes que varían cada vez que se conectan a una clase. Esto se llevó a cabo mediante técnicas como la utilización de filtros para la energía textural y aumentar o disminuir el contraste. De cada imagen se generan 40 imágenes nuevas de tal forma que se etiquetan en una clase determinada, con la finalidad de hacer una clasificación supervisada, es por eso que se agrupan en las mismas 12 clases de acuerdo a la imagen original.

Las pruebas en vivo se realizaron con 60 imágenes que se convirtieron en 2400 imágenes utilizando el proceso anterior. Estas imágenes fueron adquiridas de 60 estudiantes de la carrera de ingeniería de sistemas de la 
Universidad Francisco de Paula Santander Ocaña del segundo semestre del año 2020 y se validó la identidad al inicio del semestre por medio de la primera imagen tomada de la clase y las fotos dejadas en el sistema de admisiones de su registro personal. Antes de modificar las imágenes se debió realizar un preprocesamiento donde se eliminan ruidos de la imagen que puede deberse en su mayoría a los efectos de iluminación, cada imagen se convierte a escala de grises y se normalizan los datos.

\subsection{Extracción de características}

En la extracción de características se crean tres vectores de cinco características con distancia de 1 y grados de 0,45 y 90 obtenidos al aplicar la matriz de coocurrencia (Senthilkumar y Gnanamurthy, 2019). Una vez obtenida la matriz se extrae las características por medio de cinco descriptores de textura de Haralick (Entropía, Uniformidad, Contraste, Homogeneidad y Varianza), además se utiliza la máscara Laplaciana para realzar los bordes. Estas características son extraídas después de haber modificado la imagen mediante 5 filtros de textura de la Energía de Laws con vecindarios de 3×3, 5x5 y 7x7 para un total de 15 imágenes nuevas, los cuales permiten resaltar rizos, oscilación, forma y bordes; los cambios en el contraste se utilizaron con 20 umbrales a la imagen original y 5 imágenes nuevas donde se modifica la suavidad de la imagen, para el total de las 40 imágenes a las que se le extrae los descriptores estadísticos. Este proceso se aplica con la finalidad de prever cual cambio en la imagen al momento de la conexión y hacer más robusto el proceso de reconocimiento.

\subsection{Patrones}

De cada imagen se han obtenido 15 medidas de acuerdo a la distancia y el ángulo aplicado, donde se pueden realizar pruebas con un vector de 5 características o utilizarlo con todas las características extraídas. El tamaño de los descriptores de características es bastante reducido es por eso que no hay necesidad de reducción de dimensionalidad. Para poder hacer una clasificación supervisada se hace un entrenamiento con el $70 \%$ de las imágenes y el 30\% para prueba, resultando 28 para entrenamiento y 12 para prueba por cada clase, estos patrones obtenidos son los que se utilizan cada vez que el estudiante se conecta a una clase virtual donde deba presentar una prueba y se correlacionan utilizando la distancia Euclidea.

\section{Resultados}

A continuación se presentan los resultados de las etapas de procesamiento de las imágenes.

\subsection{Fuente de datos y preprocesamiento}

Las imágenes fueron adquiridas de la base de datos de Generated Photos (2020) de 1024x1024 pixeles, y fueron procesadas convirtiéndolas de color a escalas de grises para eliminar matiz y saturación, manteniendo la luminancia. En la figura 2 y 3 se puede comprobar el preprocesamiento a un conjunto de imágenes originales. 
Figura 2

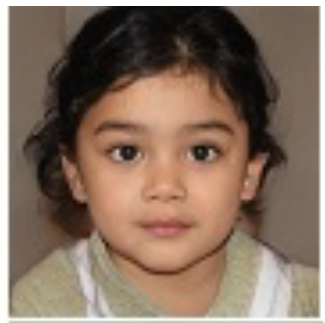

Imágenes originales
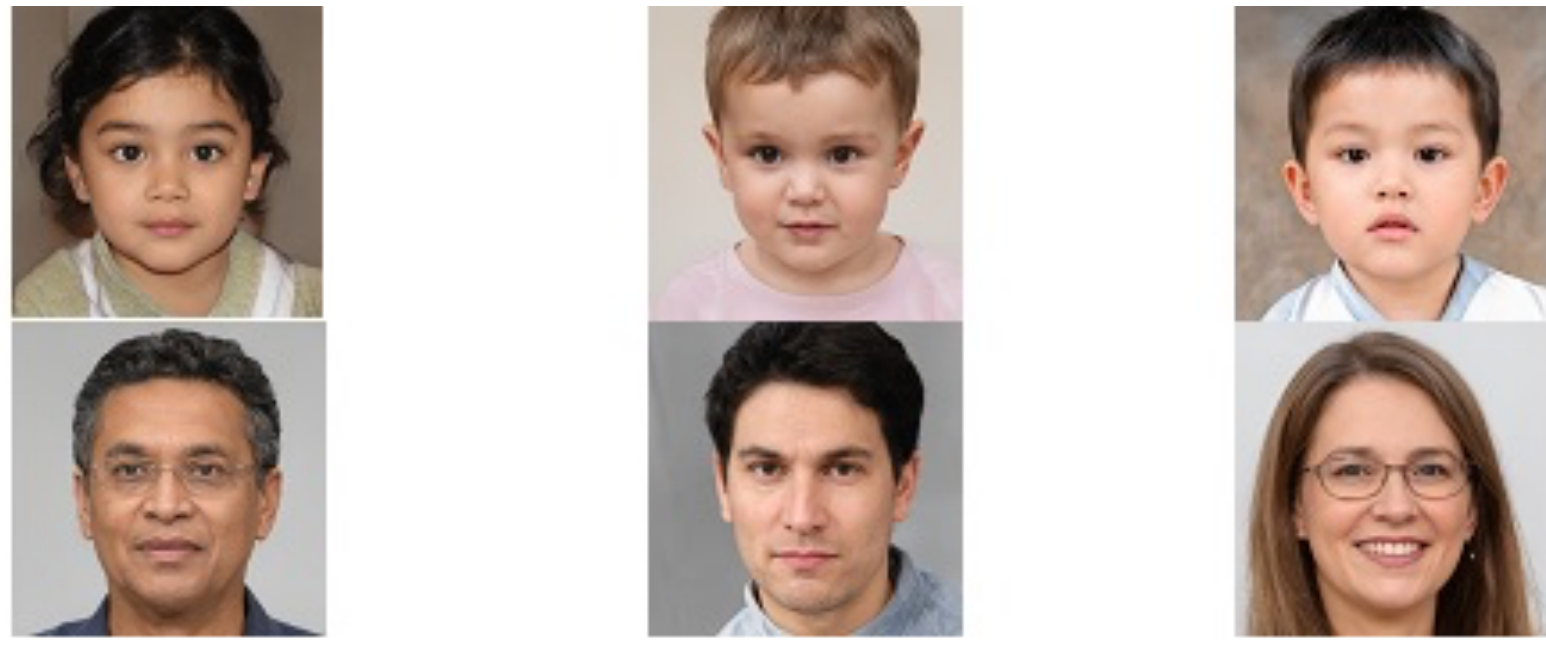

Fuente: imágenes de Generated Photos

Figura 3

Imágenes preprocesadas
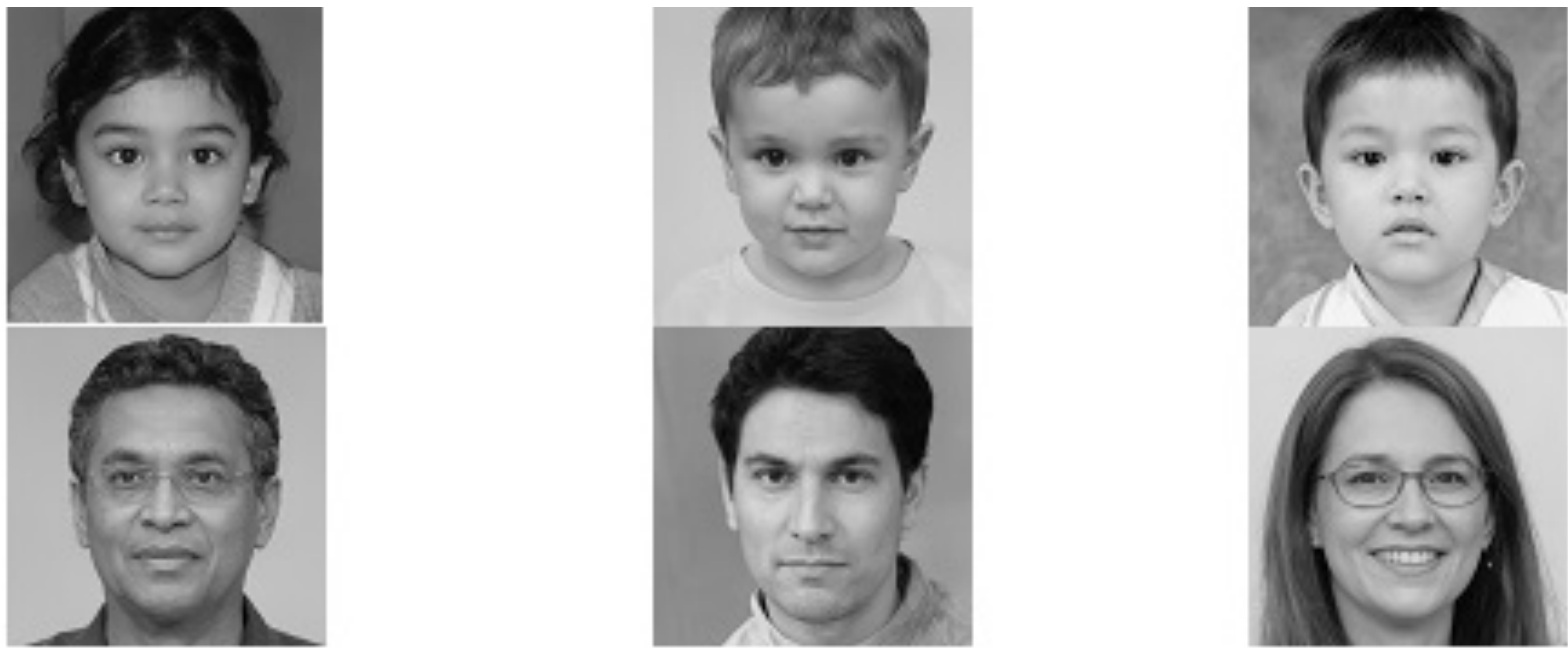

Fuente: imágenes de Generated Photos

\subsection{Exploración y transformación}

Para detectar los bordes se ha utilizado la máscara Laplaciana de una función bidimensional $f(x, y)$, la cual es una derivada de segundo orden.

$$
\nabla^{2} \mathrm{f}=\frac{\partial \mathrm{f}^{2}}{\partial \mathrm{x}}+\frac{\partial \mathrm{f}^{2}}{\partial \mathrm{x}}
$$

La máscara Laplaciana que se utilizó se muestra en el cuadro 1. 
Cuadro 1

Máscara Laplaciana

\begin{tabular}{|c|c|c|}
\hline 0 & -1 & 0 \\
\hline-1 & 4 & -1 \\
\hline 0 & -1 & 0 \\
\hline
\end{tabular}

Fuente: elaboración propia

Los descriptores de textura de Haralick fueron obtenidos de la matriz de coocurrencia (GLCM) el cual obtiene información de un par de píxeles que cumplen una condición.

$$
N_{d}(i, j)=\frac{C_{d}(i, j)}{\sum_{i} \sum_{j} C_{d}(i, j)}
$$

$C_{d}$ es una matriz de coocurrencia.

Se calcula una GLCM con direcciones 0,45 y 90, con una distancia de 1 entre sus píxeles (cuadro 2), formando 3 vectores con la extracción de resultados (figura 4).

Cuadro 2

Procesamiento de una matriz

con 0 grados y distancia de 1.

\begin{tabular}{|c|c|c|c|c|c|c|c|c|c|c|}
\hline & 0 & 1 & 2 & 3 & \multirow{5}{*}{$\rightarrow \mathrm{GLCM}$} & & 0 & 1 & 2 & 3 \\
\hline 0 & 0 & 0 & 1 & 1 & & 0 & 2 & 2 & 1 & 0 \\
\hline 1 & 0 & 0 & 1 & 1 & & 1 & 0 & 2 & 0 & 0 \\
\hline 2 & 0 & 2 & 2 & 2 & & 2 & 0 & 0 & 3 & 1 \\
\hline 3 & 2 & 2 & 3 & 3 & & 3 & 0 & 0 & 0 & 1 \\
\hline
\end{tabular}

Fuente: elaboración propia 
Figura 4

Vectores GLCM extraídos de la imagen
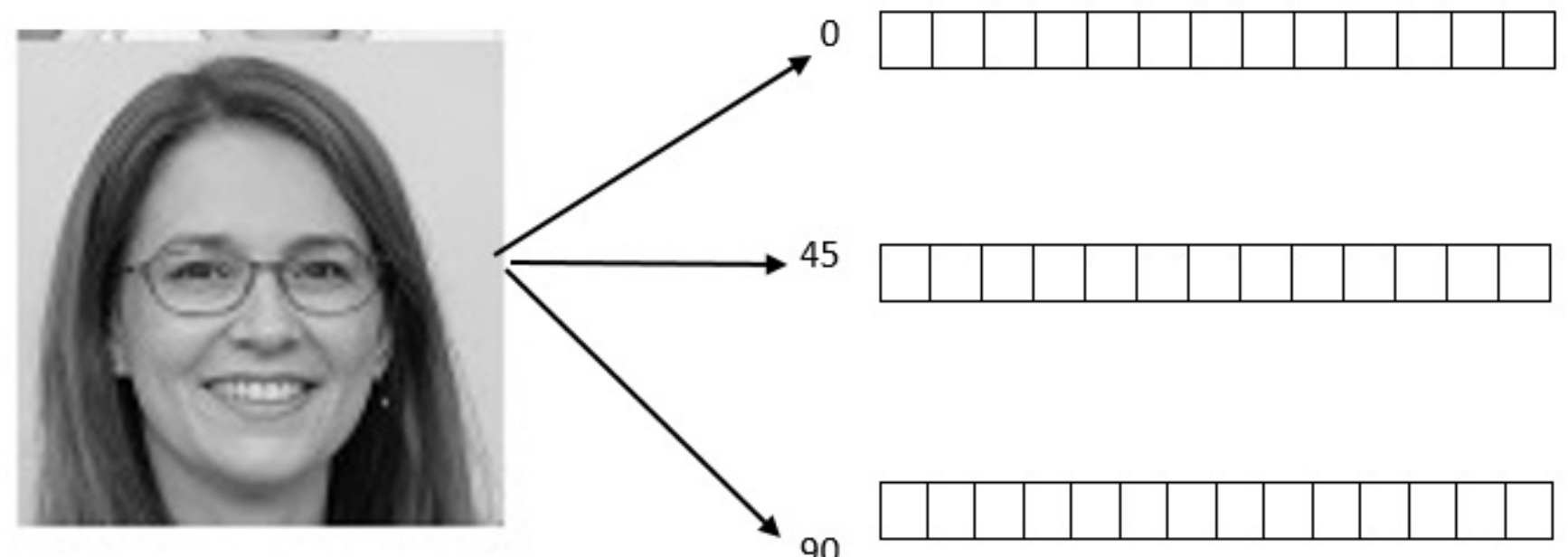

Fuente: elaboración propia (imagen de Generated Photos)

Los descriptores obtenidos fueron: Entropía, Uniformidad, Contraste, Homogeneidad y Varianza.

Descriptores de Haralick

Entropía, la información se encuentra en la diagonal principal de la matriz; $C(i, j)$ es cada elemento de la matriz.

$$
\text { Entropia }=-\sum_{i} \sum_{j} C(i, j) \log _{2}(C(i, j))
$$

Uniformidad o Segundo momento angular, brinda información sobre la suavidad de la textura.

$$
A S M=-\sum_{i} \sum_{j} C(i, j)^{2}
$$

El contraste proporciona información sobre variaciones muy elevadas en la imagen.

$$
\text { Contraste }=-\sum_{i} \sum_{j}|i-j|^{2} C(i, j)
$$

La homogeneidad aumenta el valor cuando los elementos estén más cerca de la diagonal principal.

$$
\text { Homogeneidad }=\sum_{i} \sum_{j} \frac{C(i, j)}{1+(i-j)^{2}}
$$

La varianza proporciona información de la dispersión que tiene el conjunto de muestras.

$$
\text { Varianza }=\sum_{i} \sum_{j}(i-u)^{2} C(i, j)
$$

Donde u es el valor medio

Las imágenes adquiridas son modificadas para simular distintos ambientes de iluminación u objetos existentes que pueden aparecer cuando se realizan nuevas videoconferencias (Figura 5). 
Figura 5

Imagen distorsionada

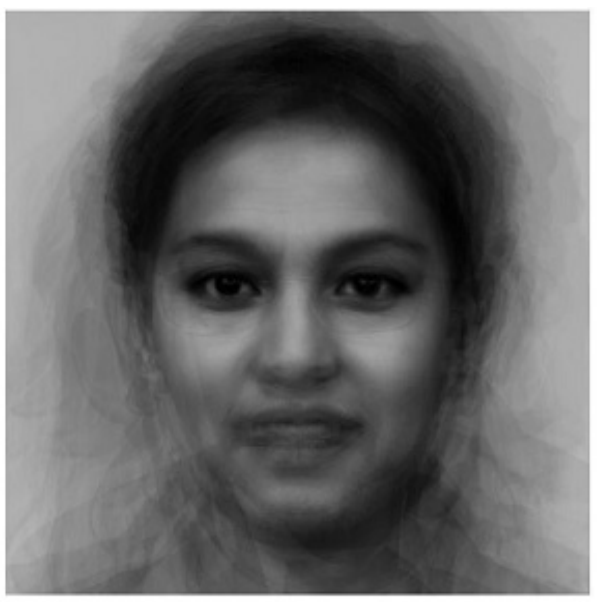

Fuente: elaboración propia

En la figura 6, se presenta una imagen reconstruida que intenta realizar un reconocimiento con imágenes adquiridas anteriormente de la misma persona que estaban en clases pasadas. La reconstrucción de imágenes puede brindar un reconocimiento eficiente para casos en los que el estudiante ha cambiado de corte de cabello o mantiene objetos en su rostro.

Figura 6

Imagen reconstruida
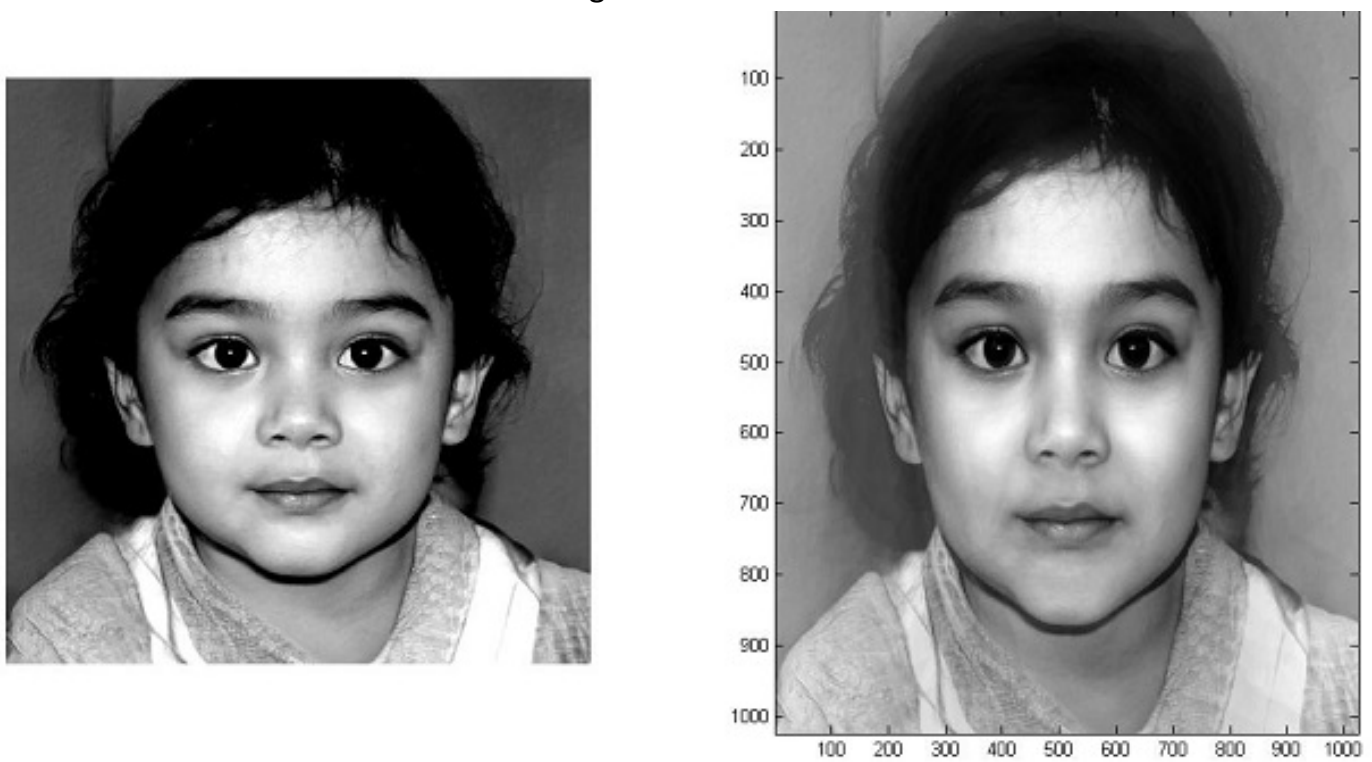

Fuente: elaboración propia

\subsection{Evaluación de descriptores}

En la evaluación de descriptores mostrados en el cuadro 3, se puede concluir que los errores de entrenamiento no predisponen a errores en las pruebas, donde la tasa de error es 7,64\%, siendo aceptable para el reconocimiento. 
Cuadro 3

Distancia 1 con 0 grados.

\begin{tabular}{|c|c|c|c|c|c|c|c|}
\hline Imagen & $E$ & $P$ & CE & CP & EE & EP & Error \\
\hline 1 & 28 & 12 & 0 & 1 & 0,00 & 8,33 & 2,5 \\
\hline 2 & 28 & 12 & 8 & 2 & 28,57 & 16,67 & 25 \\
\hline 3 & 28 & 12 & 3 & 1 & 10,71 & 8,33 & 10 \\
\hline 4 & 28 & 12 & 5 & 0 & 17,86 & 0,00 & 12,5 \\
\hline 5 & 28 & 12 & 3 & 2 & 10,71 & 16,67 & 12,5 \\
\hline 6 & 28 & 12 & 8 & 3 & 28,57 & 25,00 & 27,5 \\
\hline 7 & 28 & 12 & 9 & 1 & 32,14 & 8,33 & 25 \\
\hline 8 & 28 & 12 & 0 & 0 & 0,00 & 0,00 & 0 \\
\hline 9 & 28 & 12 & 0 & 0 & 0,00 & 0,00 & 0 \\
\hline 10 & 28 & 12 & 1 & 0 & 3,57 & 0,00 & 2,5 \\
\hline 11 & 28 & 12 & 2 & 0 & 7,14 & 0,00 & 5 \\
\hline 12 & 28 & 12 & 3 & 1 & 10,71 & 8,33 & 10 \\
\hline
\end{tabular}

$\mathrm{E}=$ entrenamiento; $\mathrm{P}=$ prueba; $\mathrm{CE}=$ mal entrenamiento; $\mathrm{CP}=$ mal prueba; $\mathrm{EE}=$ porcentaje error $\mathrm{E} ; \mathrm{EP}=$ porcentaje error prueba \%E= Porcentaje de error

Fuente: elaboración propia

En el cuadro 4, los errores descienden y junto con las pruebas se obtienen valores de 5,56\%, acercándose a valores de mayor seguridad.

\section{Cuadro 4}

Distancia 1 con 45 grados.

\begin{tabular}{|c|c|c|c|c|c|c|c|}
\hline Imagen & $E$ & $P$ & CE & CP & EE & EP & Error \\
\hline 1 & 28 & 12 & 1 & 1 & 3,57 & 8,33 & 5 \\
\hline 2 & 28 & 12 & 5 & 0 & 17,86 & 0,00 & 12,5 \\
\hline 3 & 28 & 12 & 3 & 1 & 10,71 & 8,33 & 10 \\
\hline 4 & 28 & 12 & 2 & 0 & 7,14 & 0,00 & 16,67 \\
\hline 5 & 28 & 12 & 6 & 2 & 21,43 & 20 \\
\hline 6 & 28 & 12 & 2 & 1 & 7,14 & 8,33 & 7,5 \\
\hline 7 & 28 & 12 & 1 & 2 & 3,57 & 16,67 & 7,5 \\
\hline 8 & 28 & 12 & 0 & 0 & 0,00 & 0,00 & 0 \\
\hline 9 & 28 & 12 & 0 & 0 & 0,00 & 0,00 & 0 \\
\hline 10 & 28 & 12 & 1 & 1 & 3,57 & 8,33 & 5 \\
\hline 11 & 28 & 12 & 2 & 0 & 7,14 & 0,00 & 5 \\
\hline 12 & 28 & 12 & 0 & 0 & 0,00 & 0,00 & 0 \\
\hline
\end{tabular}

$\mathrm{E}=$ entrenamiento; $\mathrm{P}=$ prueba; $\mathrm{CE}=$ mal entrenamiento; $\mathrm{CP}=$ mal prueba; $\mathrm{EE}=$ porcentaje error $\mathrm{E} ; \mathrm{EP}=$ porcentaje error prueba $\% \mathrm{E}=$ Porcentaje de error

Fuente: elaboración propia

En la tabla 5, cuando se analizan con 90 grados la tasa de error es del 4,86 demostrando la efectividad del método, y la conveniencia de utilizar la distancia de 1 y 90 grados. 
Cuadro 5

Distancia uno con 90 grados.

\begin{tabular}{|c|c|c|c|c|c|c|c|}
\hline Imagen & $E$ & $P$ & $\mathrm{CE}$ & $\mathrm{CP}$ & $\mathrm{EE}$ & EP & Error \\
\hline 1 & 28 & 12 & 0 & 1 & 0,00 & 8,33 & 2,5 \\
\hline 2 & 28 & 12 & 4 & 0 & 14,29 & 0,00 & 10 \\
\hline 3 & 28 & 12 & 3 & 1 & 10,71 & 8,33 & 10 \\
\hline 4 & 28 & 12 & 1 & 1 & 3,57 & 8,33 & 5 \\
\hline 5 & 28 & 12 & 3 & 1 & 10,71 & 8,33 & 10 \\
\hline 6 & 28 & 12 & 1 & 1 & 3,57 & 8,33 & 5 \\
\hline 7 & 28 & 12 & 4 & 1 & 14,29 & 8,33 & 12,5 \\
\hline 8 & 28 & 12 & 2 & 0 & 7,14 & 0,00 & 5 \\
\hline 9 & 28 & 12 & 0 & 0 & 0,00 & 0,00 & 0 \\
\hline 10 & 28 & 12 & 1 & 0 & 3,57 & 0,00 & 2,5 \\
\hline 11 & 28 & 12 & 0 & 0 & 0,00 & 0,00 & 0 \\
\hline 12 & 28 & 12 & 0 & 1 & 0,00 & 8,33 & 2,5 \\
\hline \multicolumn{8}{|c|}{$\begin{array}{c}\mathrm{E}=\text { entrenamiento; } \mathrm{P}=\text { prueba; } \mathrm{CE}=\text { mal entrenamiento; } \mathrm{CP}=\text { mal prueba; } \mathrm{EE}=\text { porcentaje error } \mathrm{E} ; \mathrm{EP}=\text { porcentaje erro } \\
\text { prueba } \% \mathrm{E}=\text { Porcentaje de error }\end{array}$} \\
\hline
\end{tabular}

Fuente: elaboración propia

En el gráfico 1, se puede evidenciar que los datos de error están por debajo del $25 \%$ en el peor de los casos, logrando tasas de acierto del $90 \%$. El error total es de 7,64\%, con un 5,41\% al utilizar 90 grados. Los resultados permiten evidenciar que el método puede ser usado en reuniones de videoconferencias, con participantes en diferentes ambientes y cambios en su imagen, lo cual permitirá llevar un mayor control por parte del docente, estudiantes y padres de familia. El sistema puede ser incorporado para extraer imágenes en tiempo real de cada uno de los participantes y compararlos para un efectivo reconocimiento.

Todo el proceso anterior es realizado en otra prueba con 60 imágenes de clases en vivo, y sin intentar engañar al sistema, logrando resultados de reconocimiento del $100 \%$.

\section{Gráfico 1}

Porcentajes de error con 1, 45 y 90 grados.

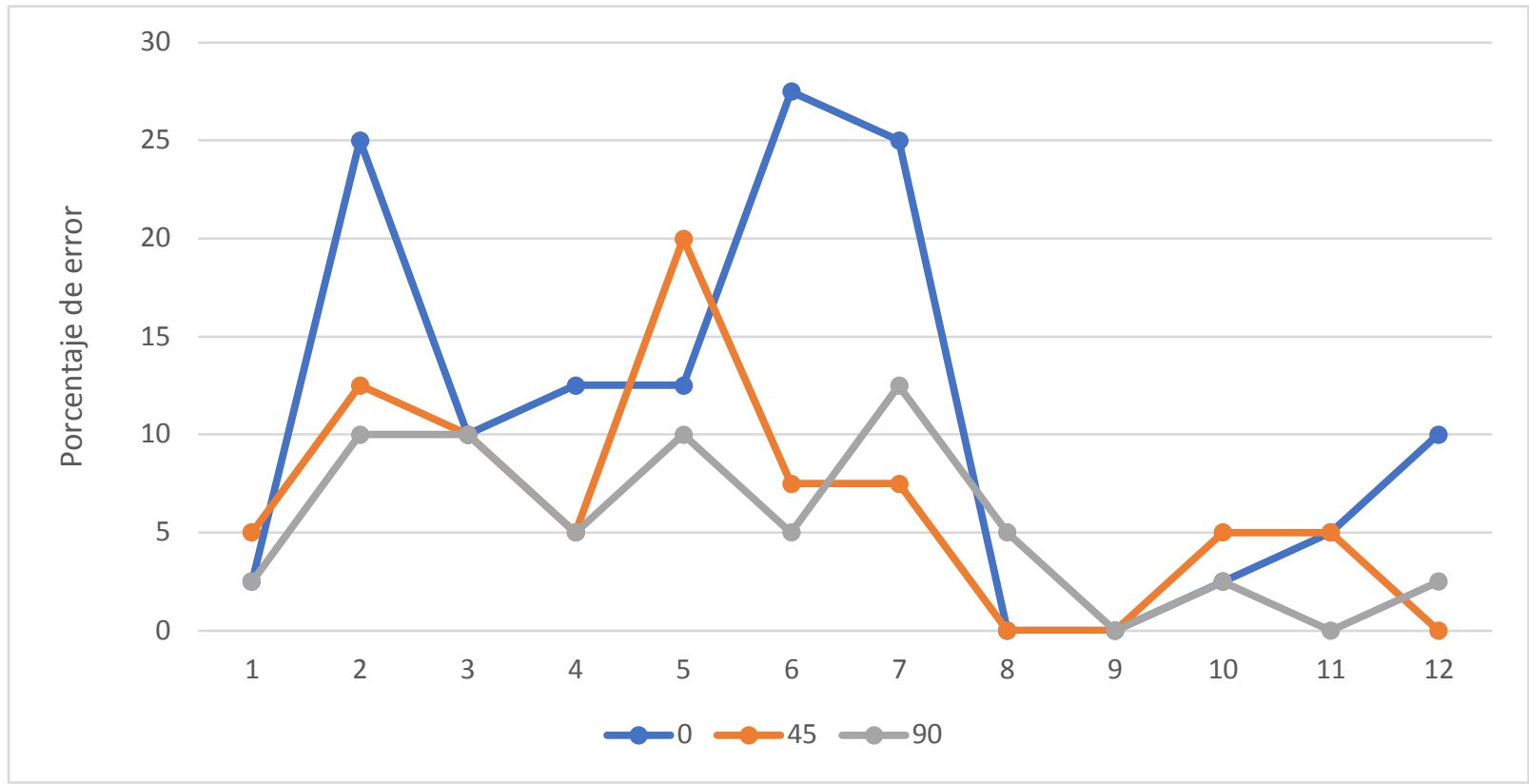

Fuente: elaboración propia 


\subsection{Discusión}

A continuación se hace una discusión de las ventajas y desventajas al comparar la propuesta con los métodos más utilizados para la extracción y descripción de características como son SIFT y SURF.

La extracción de características permite hacer un reconocimiento más preciso de una imagen pero también aumenta el tiempo de procesamiento si contiene un elevado número de descriptores; los métodos SIFT y SURF presentan este inconveniente debido a la cantidad de puntos de interés que pueden detectar, esto se debe a que el método SIFT aplica un filtro Gaussiano, luego hace una diferencia de Gaussianas y detecta puntos de interés con un re-escalado de la imagen, lo cual genera una cantidad de puntos en cada una de las imágenes con diferentes valores tal como lo muestra la figura 7, donde inclusive se puede llegar a tener más de 50 mil puntos de interés para una clasificación.

El método SURF hace una detección rápida mejorando casi en un 300\% la obtención de las características sin embargo sigue siendo alto. Este problema debe ser solucionado aplicando una reducción de redimencionalidad basada en un análisis de componentes principales (PCA) el cual entrega los resultados mostrados en el cuadro 6, donde se puede ver que después del PCA sigue siendo alto el número de descriptores para el reconocimiento de una imagen, al compararlo con el método propuesto el cual solo necesita de 5 descriptores, esto aumentara el tiempo de proceso del SIFT y el SURF en más de 13 veces comparado con los descriptores de textura. Al hablar del tipo de clasificador utilizado por SIFT y SURF debido a la cantidad de descriptores se utilizan clasificadores sencillos como Knn o SVM pero más complejos que la distancia Euclidea.

Figura 7

Imagen distorsionada

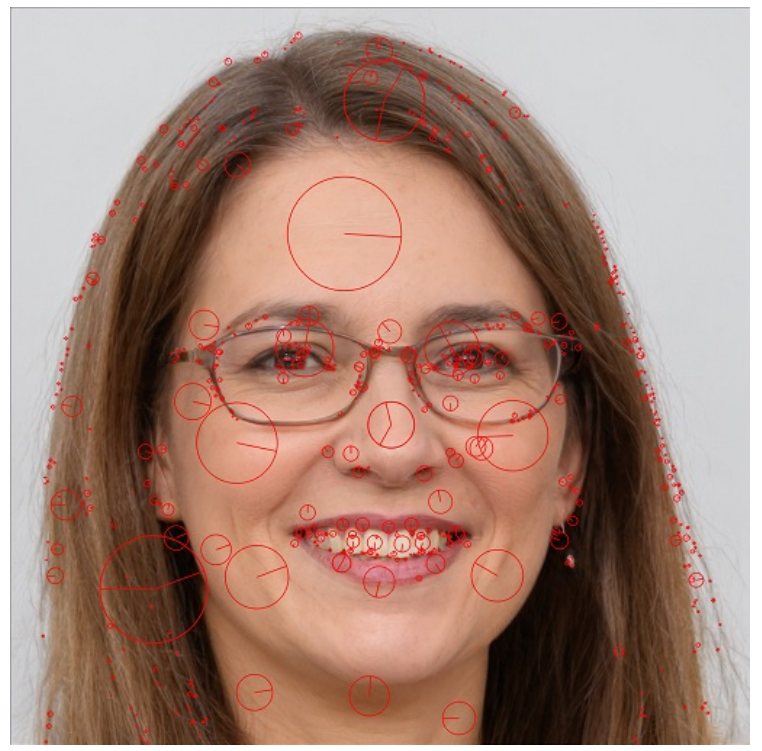

Fuente: elaboración propia

Otra desventaja de SIFT y SURF es que representan la imagen mediante un conjunto de puntos los cuales son necesarios combinar mediante estrategias de fusión, que podría incrementar la cantidad de puntos de interés. En el cuadro 7, se observa que el método propuesto solo necesita una estrategia de fusión a nivel de descripción cuando se combina con diferentes descriptores estadísticos de textura, mientras que SIFT y SURF debe utilizarlos también a nivel de representación y de decisión. 


\section{Cuadro 6}

Dimencionalidad después de

aplicar PCA al SIFT y SUF

\begin{tabular}{|c|c|c|c|}
\hline Detector y descriptor & Representación de la imagen & Clasificación & Dimensión \\
\hline SIFT & Conjunto de puntos & Knn - SVM & 328 \\
\hline SURF & Conjunto de puntos & Knn - SVM & 64 \\
\hline Método propuesto & Descriptores de textura & Distancia Euclidea & 5 \\
\hline
\end{tabular}

Fuente: elaboración propia

Cuadro 7

Estrategias de fusión

\begin{tabular}{|c|c|c|c|}
\hline Detector y descriptor & A nivel de descripción & A nivel de representación & A nivel de decisión \\
\hline SIFT & $\mathrm{X}$ & $\mathrm{X}$ & $\mathrm{X}$ \\
\hline SURF & $\mathrm{X}$ & $\mathrm{X}$ & $\mathrm{X}$ \\
\hline Método propuesto & $\mathrm{X}$ & & \\
\hline
\end{tabular}

Fuente: elaboración propia

En cuanto a la implementación, el método de descriptores de textura puede ser fácilmente implementado en lenguajes como Matlab o Python que cuentan con funciones básicas ya implementadas en estos lenguajes para la adquisición y procesamiento de imágenes, mientras que SIFT y SURF son algoritmos más complejos y es necesario tener conocimientos avanzadas de programación y procesamiento de imágenes.

La desventaja que presenta el método de descriptores de textura es que puede implementarse para reconocimiento de personas con grupos reducidos tales como una clase virtual, la cual puede presentar máximo una cantidad de 100 a 250 estudiantes, pero para una mayor población la descripción de la textura total de una imagen podría no dar buenos resultados; mientras que SIFT y SURF pueden ser utilizados de forma robusta y con bases de datos grandes en la búsqueda de personas sin realizar un entrenamiento supervisado. Los resultados de error con SIFT son de 9,67\%, con SURF de 6,93\% y con descriptores de textura de $7.64 \%$, y los 3 tienen un porcentaje de acierto del $100 \%$ cuando se realizó pruebas en línea; aunque se observa que SURF es relativamente más preciso la diferencia no es determinante debido a las desventajas que puede presentar en tiempo de procesamiento y estrategias de fusión.

Para la detección de suplantación de estudiantes en clases virtuales usando el reconocimiento de rostros el método de textura puede ser utilizado de forma más eficaz, por su menor tiempo de procesamiento y cantidad de descriptores además de la facilidad de implementación.

\section{Conclusiones}

Los descriptores de Haralick pueden ser utilizados para el reconocimiento de personas en videoconferencias con ambientes modificados. Logrando tasas de acierto del 100\% en línea y de 92,36 en cambios de ambiente al modificar las imágenes. Con lo cual se puede prevenir ataques o ingresos no permitidos en las clases que se imparten de forma virtual, ayudando a prevenir el fraude y permitiendo tomar acciones de forma inmediata. El método reduce el tiempo de procesamiento y la cantidad de descriptores a utilizar, así como una mejor implementación. Con la llegada del COVID-19 el método puede ofrecer una ayuda importante para el reconocimiento de estudiantes con perfiles falsos en salas virtuales donde la población es reducida; no se han realizado pruebas para otras tareas de reconocimiento donde el método podría funcionar de forma adecuada. 


\section{Referencias bibliográficas}

Agarwal, A., Singh, R., y Vatsa, M. (2016). Face anti-spoofing using Haralick features. 2016 IEEE 8th International Conference on Biometrics Theory, Applications and Systems (BTAS), 1-6. https://doi.org/10.1109/BTAS.2016.7791171

Alazawi, S. A., Shati, N. M., y Abbas, A. H. (2019). Texture features extraction based on GLCM for face retrieval system. Periodicals of Engineering and Natural Sciences (PEN), 7(3), 1459. https://doi.org/10.21533/pen.v7i3.787

Ali Khan, S., Hussain, A., y Usman, M. (2016). Facial expression recognition on real world face images using intelligent techniques: A survey. Optik, 127(15), 6195-6203. https://doi.org/10.1016/j.ijleo.2016.04.015

Alvez, C. E., Benedetto, M. G., Etchart, G. R., Luna, L. J., Leal, C. R., Fernández, M. A., ... Loggio, S. R. (2014). PID 7035 Identificación de personas mediante Sistemas Biométricos. Estudio de factibilidad y su implementación en organismos estatales. Ciencia, Docencia y Tecnología Suplemento, 4(4), 48-71.

Beltrán, L. C. (2017). La videoconferencia como herramienta en el e-learning con los estudiantes de la especialización en docencia universitaria de la Universidad Cooperativa de Colombia. 4to Congreso Internacional AmITIC 2017. Recuperado de https://core.ac.uk/reader/234021120

Bravo-Mancero, J., Larrea, C., Ruales, R., y Cruz, J. (2020). Covid 19: De la educación tradicional y alfabetización de adultos al uso de dispositivos para el inter-aprendizaje. Brazilian Journal of Health Review, 3, 46664682. https://doi.org/10.34119/bjhrv3n3-059

Cáceres-Piñaloza, K. F. (2020). Educación virtual: Creando espacios afectivos, de convivencia y aprendizaje en tiempos de COVID-19. CienciAmérica, 9(2), 38-44. https://doi.org/10.33210/ca.v9i2.284

Ding, X. (2017). Texture Feature Extraction Research Based on GLCM-CLBP Algorithm. 7th International Conference on Education, Management, Information and Mechanical Engineering (EMIM 2017). Atlantis Press.

Friedman, A., Blau, I., y Eshet-Alkalai, Y. (2016). Cheating and Feeling Honest: Committing and Punishing Analog versus Digital Academic Dishonesty Behaviors in Higher Education. Interdisciplinary Journal of e-Skills and Lifelong Learning, 12, 193-205. https://doi.org/10.28945/3629

Gámez, F. D. G., y Agapito, J. B. (2016). Autenticación facial como soporte extra en los entornos virtuales de aprendizaje para evitar el fraude académico. Revista Tecnología, Ciencia y Educación, O(3). Recuperado de https://www.revistasocitec.org/index.php/TCE/article/view/63

Jia, S., Guo, G., y Xu, Z. (2020). A survey on 3D mask presentation attack detection and countermeasures. Pattern Recognition, 98, 107032. https://doi.org/10.1016/j.patcog.2019.107032

Karami, E., Shehata, M., y Smith, A. (2015, noviembre 5). Image Identification Using SIFT Algorithm: Performance Analysis against Different Image Deformations.

Kushwaha, R., y Nain, N. (2020). PUG-FB: Person-verification using geometric and Haralick features of footprint biometric. Multimedia Tools and Applications, 79(3), 2671-2701. https://doi.org/10.1007/s11042-01908149-0

Neeru, N., y Kaur, L. (2016, febrero 11). Modified SIFT Descriptors for Face Recognition under Different Emotions [Research Article]. https://doi.org/10.1155/2016/9387545 
Núñez-Cortés, J. M., Reussi, R., García Dieguez, M., y Falasco, S. (2020). COVID-19 y la educación médica, una mirada hacia el futuro. Foro Iberoamericano de Educación Médica (FIAEM). Educación Médica, 21(4), 251258. https://doi.org/10.1016/j.edumed.2020.06.004

Oyallon, E., y Rabin, J. (2015). An Analysis of the SURF Method. Image Processing On Line, 5, 176-218. https://doi.org/10.5201/ipol.2015.69

Senthilkumar, D., y Gnanamurthy, R. (2019). HANFIS: A New Fast and Robust Approach for Face Recognition and Facial Image Classification. https://doi.org/10.1007/978-981-10-8968-8_8

Vidal Martínez, A. A., y Camarena Gómez, B. O. (2015). Evolución y análisis de una experiencia de utilización de videoconferencia de sala y de escritorio. Píxel-Bit, Revista de Medios y Educación, (47), 59-71. https://doi.org/10.12795/pixelbit.2015.i47.04

Vinay, A., Hebbar, D., Shekhar, V. S., Murthy, K. N. B., y Natarajan, S. (2015). Two Novel Detector-Descriptor Based Approaches for Face Recognition Using SIFT and SURF. Procedia Computer Science, 70, 185-197. https://doi.org/10.1016/j.procs.2015.10.070

Esta obra está bajo una Licencia Creative Commons Attribución-NoCommercial 4.0 International

(cc) BY-NC 